\title{
INVARIANTS OF PFAFFIAN SYSTEMS $\left({ }^{1}\right)$
}

\author{
BY \\ ROBERT B. GARDNER
}

1. Introduction. Let $M$ be a real $C^{\infty}$-differentiable manifold, and $T^{*}(M)$ its cotangent bundle. Let $c(M)$ denote the ring of $C^{\infty}$-functions on $M$, and let $\Gamma^{*}(M)$ denote the $c(M)$-module of global $C^{\infty}$-sections of $T^{*}(M)$.

Definition 1. A Pfaffian system is a $c(M)$-submodule of $\Gamma^{*}(M)$.

Dually we let $T(M)$ denote the tangent bundle and $\Gamma(M)$ the $c(M)$-module of global $C^{\infty}$-sections of $T(M)$.

Definition 2. A vector field system is a $c(M)$-submodule of $\Gamma(M)$.

Let $I$ be a Pfaffian system, and let $U$ be an open neighborhood on $M$, then we let $I_{U}$ denote the $c(U)$-module obtained by the restriction of the one-forms in $I$ to $U$.

Definition 3. A Pfaffian system is said to have dimension $n$ at a point $p \in M$ if there exists a neighborhood $U$ of $p$ and $n$-linearly independent one-forms defined on $U$ which generate $I_{U}$.

Definition 4. A Pfaffian system is said to be nonsingular if the dimension is defined at every point $p \in M$ and is constant.

In this paper we will assume that all Pfaffian systems under consideration are nonsingular.

In $\$ 2$ we review in a modern formulation the notions of class, derived systems, and type; and we conclude with a set of integrability conditions for derived systems. In $\$ 3$ we consider the rank of a Pfaffian system and prove a generalization of the theorem of Cartan-von Weber. In $\S 4$ we introduce a conformal tensor defined on Pfaffian systems with even codimension, and a conformal tensor on the second derived system of Pfaffian systems satisfying a particular type restriction. In the last two chapters we analyze the cases when the conformal tensors introduced in $\$ 4$ become conformal bilinear forms. As an application we consider the Pfaffian system associated with a second order partial differential equation in one independent and two dependent variables. This leads to a new interpretation of the type and characteristic curves of such equations. We conclude the paper by deducing certain invariants of the second derived system of normal systems of codimension two and by proving an integrability theorem.

2. The type. Let $E\left(\Gamma^{*}(M)\right)$ denote the exterior algebra over $\Gamma^{*}(M)$, and let

Received by the editors December 27, 1965 .

(') The results in this paper were partially obtained at the Courant Institute of Mathematical Sciences, New York University, under the sponsorship of the National Science Foundation, Grant NSF-GP-3465. 
\rfloor denote the interior product adjoint to left exterior multiplication on $E(\Gamma(M))$, and let

$$
d: E\left(\Gamma^{*}(M)\right) \rightarrow E\left(\Gamma^{*}(M)\right)
$$

denote the exterior derivative on $M$.

Given a Pfaffian system $I$, we may associate a vector field system

$$
\text { Char } I=\{X \in \Gamma(M) \mid X\lrcorner w=0, X\lrcorner d w \in I \text {, for all } w \in I\}
$$

called the characteristic system of $I$, which is known to be completely integrable [10].

The annihilator of the characteristic system is a Pfaffian system

$$
C(I)=[\operatorname{Char} I]^{\perp}
$$

called the Cartan system of $I$. The basic property of this system is given by the following theorem of E. Cartan [5].

Theorem 1. Let I be a Pfaffian system, then the Cartan system of I is the smallest completely integrable Pfaffian system with the property that if $\left\{x^{1}, \ldots, x^{p}\right\}$ is a local system of first integrals, then locally there exist generators for I which only depend on $\left\{x^{1}, \ldots, x^{p}\right\}$ and their differentials.

Definition 5. The dimension of the Cartan system of a Pfaffian system $I$ is called the class of $I$.

The class, therefore, is the minimum number of variables necessary in order to write down local generators of the system.

Let $I$ be a Pfaffian system, then $I$ is a $c(M)$-submodule of the $c(M)$-module $\Gamma^{*}(M)$, and we may form the quotient module

$$
\Gamma^{*}(M) / I \text {. }
$$

The space of exterior two-forms over this space is again a $c(M)$-module and we introduce the notation

$$
\Lambda^{2}\left(\Gamma^{*}(M) / I\right)=\Lambda^{2} \Gamma^{*}(M) \bmod I .
$$

The composition $\delta$ of exterior differentiation with the natural projection

$$
I \stackrel{d}{\longrightarrow} \Lambda^{2} \Gamma^{*}(M) \longrightarrow \Lambda^{2} \Gamma^{*}(M) \bmod I
$$

is a $c(M)$-module homomorphism. Thus the image of $I$ under $\delta$ is a $c(M)$-module which we denote by $d I \bmod I$, and the kernel of $\delta$ is a $c(M)$-submodule of $I$ which we denote by $I^{(1)}$. The submodule $I^{(1)}$ is called the first derived system of $I$, and we have a short exact sequence of $c(M)$-modules

$$
0 \longrightarrow I^{(1)} \longrightarrow I \stackrel{\delta}{\longrightarrow} d I \bmod I \longrightarrow 0 \text {. }
$$


Definition 6. Let $I$ be a Pfaffian system, the $r$ th derived system $I^{(r)}$ is defined inductively by

$$
0 \longrightarrow I^{(r)} \longrightarrow I^{(r-1)} \stackrel{\delta}{\longrightarrow} d I^{(r-1)} \bmod I^{(r-1)} \longrightarrow 0 .
$$

An immediate consequence of the definition is the existence of a smallest nonnegative integer $N$ such that either

$$
I^{(N+p)}=\{0\} \quad \text { or } \quad I^{(N+p)}=I^{(N+p+1)}
$$

for all $p \geqq 0$. We note that $I^{(N)}$ is the largest completely integrable system contained in $I$.

Definition 7. Let $I$ be a Pfaffian system, then the smallest nonnegative integer $N$ which satisfies (1) is called the derived length of $I$.

Thus if $I$ is a Pfaffian system of derived length $N$, there is an increasing sequence of $c(M)$-modules

$$
I^{(N)} \subset \cdots \subset I^{(1)} \subset I,
$$

and assuming that these are all nonsingular we may define integers by

$$
p_{0}=\operatorname{dim} I^{(N)} ; \operatorname{dim} I^{(i)} / I^{(i+1)}=p_{N-i} \quad(0 \leqq i \leqq N-1) .
$$

As a result

$$
\operatorname{dim} I=\sum_{i=0}^{N} p_{i},
$$

and if we let $p_{N+1}$ denote the codimension of $I$ in $C(I)$,

$$
\text { class } I=\sum_{i=0}^{N+1} p_{i} .
$$

Matters being so, we say that the Pfaffian system $I$ has type

$$
\left(p_{0}, \ldots, p_{N}, p_{N+1}\right)
$$

and by abus de langage we will call $p_{N+1}$ the codimension of $I$.

The type of a Pfaffian system is not arbitrary, a fact which is exhibited by the following proposition.

Proposition 1. Let I be a Pfaffian system of type

$$
\left(p_{0}, \ldots, p_{N}, p_{N+1}\right)
$$

then for $-1 \leqq i \leqq N-1$,

$$
P_{N-i-1} \leqq p_{N-i}\left(p_{N-i+1}+p_{N-i+2}+\cdots+p_{N}+p_{N+1}\right)+\left(\begin{array}{c}
p_{N-i} \\
2
\end{array}\right) \text {. }
$$

Proof. Let $\left\{\phi^{1}, \ldots, \phi^{m}\right\}$ define a basis of $I^{(i+1)}$, and let $\left\{\beta^{1}, \ldots, \beta^{p_{N}-1}\right\}$ define a basis of a complement of $I^{(i+1)}$ in $I^{(i)}$, then we will have formulas

$$
d \phi^{k} \equiv \sum \beta^{j} \wedge \eta_{j}^{k} \bmod I^{(i+1)}
$$


and hence

$$
\operatorname{dim} I^{(i+1)} / I^{(i+2)} \leqq \operatorname{dim} I^{(i)} / I^{(i+1)} \cdot \operatorname{dim} C(I) / I^{(i)}+\operatorname{dim} \Lambda^{2} I^{(i)} / I^{(i+1)},
$$

which gives the above inequality.

In particular if $I$ is an $n$-dimensional Pfaffian system of class $n+m$, then these inequalities imply

$$
\operatorname{dim} I^{(1)} \geqq n-m(m-1) / 2 .
$$

Proposition 2. Let I be a Pfaffian system, and let $\left\{\alpha^{1}, \ldots, \alpha^{p_{N}-r}\right\}$ define a basis for any complement of $I^{(r+1)}$ in $I^{(r)}$, then for all $\phi \in I^{(r+1)}$

$$
d \phi \wedge d\left(\alpha^{1} \wedge \cdots \wedge \alpha^{\left.P_{N-r}\right)} \equiv 0 \bmod I^{(r+1)} .\right.
$$

We call these equations the integrability conditions for $I^{(r+1)}$ in $I^{(r)}$.

Proof. It suffices to verify the integrability conditions for $I^{(1)}$ in $I$. Let $\left\{w^{1}, \ldots, w^{\text {s }}\right\}$ define a basis of $I^{(1)}$, and $\left\{\alpha^{1}, \ldots, \alpha^{P_{N}}\right\}$ define a basis for a complement of $I^{(1)}$ in $I$. Then by definition of $I^{(1)}$

$$
d w^{i} \wedge w^{1} \wedge \cdots \wedge w^{s} \wedge \alpha^{1} \wedge \cdots \wedge \alpha^{p_{N}}=0 \quad(1 \leqq i \leqq s)
$$

and by linear independence

$$
w^{1} \wedge \cdots \wedge w^{s} \wedge \alpha^{1} \wedge \cdots \wedge \alpha^{p_{N}} \neq 0 .
$$

Now if $\Omega$ is any exterior two-form and $\eta^{1}, \ldots, \eta^{n}$ are linearly independent oneforms, then $\Omega^{q} \wedge \eta^{1} \wedge \cdots \wedge \eta^{n}=0$ implies

$$
\Omega^{q+1} \wedge \eta^{1} \wedge \cdots \wedge \hat{\eta}^{i} \wedge \cdots \wedge \eta^{n}=0 \quad(1 \leqq i \leqq n),
$$

where the ${ }^{\wedge}$ stands for delete. Therefore (4) and (5) imply

$$
\left(d w^{i}\right)^{2} \wedge w^{1} \wedge \cdots \wedge \widehat{w}^{i} \wedge \cdots \wedge w^{s} \wedge \alpha^{1} \wedge \cdots \wedge \alpha^{p_{N}}=0
$$

for $1 \leqq i \leqq s$.

If $1 \leqq i, j \leqq s$, then by definition we have

$$
\left(d w^{i}+d w^{j}\right) \wedge w^{1} \wedge \cdots \wedge w^{s} \wedge \alpha^{1} \wedge \cdots \wedge \alpha^{p_{N}}=0
$$

and hence

$$
\left(d w^{i}+d w^{j}\right)^{2} \wedge w^{1} \wedge \cdots \wedge \widehat{w}^{j} \wedge \cdots \wedge w^{s} \wedge \alpha^{1} \wedge \cdots \wedge \alpha^{p_{N}}=0
$$

but $\left(d w^{i}+d w^{j}\right)^{2}=\left(d w^{i}\right)^{2}+2 d w^{i} \wedge d w^{j}+\left(d w^{j}\right)^{2}$ and taking account of (6), we have

$$
2 d w^{i} \wedge d w^{j} \wedge w^{1} \wedge \cdots \wedge \widehat{w}^{j} \wedge \cdots \wedge w^{s} \wedge \alpha^{1} \wedge \cdots \wedge \alpha^{p_{N}}=0 .
$$

Now by (6) and (7) the exterior derivative of (1) becomes

$$
d w^{i} \wedge w^{1} \wedge \cdots \wedge w^{s} \wedge d\left(\alpha^{1} \wedge \cdots \wedge \alpha^{p_{N}}\right)=0
$$

and, since this is true for $\left\{w^{1}, \ldots, w^{s}\right\}$ a basis of $I^{(1)}$, we have (3) as claimed. 
3. The rank. We now consider another integer valued invariant of a Pfaffian system which Cartan attributes to Engel [5].

Definition 8. Let $I$ be a Pfaffian system, then $I$ is said to have rank $\rho$ if $\rho$ is the smallest integer such that

$$
(\lambda)^{\rho+1}=0
$$

for all $\lambda \in d I \bmod I$.

Proposition 3. Let I be an n-dimensional Pfaffian system with type

$$
\left(p_{0}, \ldots, p_{N}, p_{N+1}\right)
$$

and rank $\rho$, then

$$
2 \rho \leqq p_{N+1} \leqq \rho(n+1) .
$$

Proof. This is a tautology of the elementary inequality due to Dearborn [7], which states

$$
n+2 \rho \leqq \text { class } I \leqq n+n \rho+\rho .
$$

Proposition 4. Let I be a Pfaffian system with type $\left(p_{0}, \ldots, p_{N}, p_{N+1}\right)$ then

$$
\rho\left(I^{(r)}\right) \leqq p_{N-r+1} \quad(1 \leqq r \leqq N-1) .
$$

Proof. Let $\left\{w^{1}, \ldots, w^{p_{N-r+1}}\right\}$ define a complement of $I^{(r)}$ in $I^{(r-1)}$, then for $\alpha \in I^{(r)}$ the defining relations are

$$
d \alpha \wedge w^{1} \wedge \cdots \wedge w^{p_{N-r+1}} \equiv 0 \bmod I^{(r)},
$$

and since $\left\{w^{1}, \ldots, w^{p_{N-r+1}}\right\}$ are linearly independent this implies

$$
(d \alpha)^{p_{N-r+1}} \equiv 0 \quad \bmod I^{(r)},
$$

which verifies the proposition.

TheOREM 2. Let I be a Pfaffian system with type $\left(p_{0}, \ldots, p_{N}, p_{N+1}\right)$, then

$$
(\rho(I)) \geqq p_{N}+1
$$

implies

$$
\rho\left(I^{(1)}\right) \leqq p_{N}-1 .
$$

Proof. Let $\left\{\alpha^{1}, \ldots, \alpha^{p_{N}}\right\}$ define a basis for any complement of $I^{(1)}$ in $I$, and let $\phi \in I^{(1)}$, then the defining relations give

$$
d \phi \wedge \alpha^{1} \wedge \cdots \wedge \alpha^{p_{N}} \equiv 0 \bmod I^{(1)} .
$$

Therefore, there exist one-forms $\eta_{i}$ such that

$$
d \phi \equiv \sum_{i=1}^{p_{N}} \eta_{i} \wedge \alpha^{i} \bmod I^{(1)}
$$


The integrability conditions of $I^{(1)}$ in $I$ gives

$$
d \phi \wedge d\left(\alpha^{1} \wedge \cdots \wedge \alpha^{p_{N}}\right) \equiv 0 \bmod I^{(1)}
$$

which with (8) implies

$$
\sum_{i=1}^{p_{N}}(-1)^{i+1} \eta_{i} \wedge d \alpha^{i} \equiv 0 \bmod I .
$$

As a result, for $1 \leqq j \leqq p_{N}$,

$$
d \alpha^{j} \wedge \eta_{1} \wedge \cdots \wedge \eta_{p_{N}} \equiv 0 \bmod I
$$

which if

$$
\eta_{1} \wedge \cdots \wedge \eta_{p_{N}} \not \equiv 0 \bmod I
$$

implies

$$
\left(d \alpha^{j}\right)^{p_{N}+1} \equiv 0 \bmod I .
$$

Therefore, the hypothesis that $\rho(I) \geqq p_{N}+1$ implies

$$
\eta_{1} \wedge \cdots \wedge \eta_{p_{N}} \equiv 0 \bmod I,
$$

and hence by (8)

$$
(d \phi)^{p_{N}} \equiv 0 \quad \bmod I^{(1)},
$$

but we chose $\phi \in I^{(1)}$ arbitrarily, proving $\rho\left(I^{(1)}\right) \leqq p_{N}-1$.

As a corollary we have the theorem of Cartan-von Weber [2].

COROLlARY 1. Let I be an n-dimensional Pfaffian system whose type satisfies

$$
p_{N}=1 \text {, }
$$

then if $\left\{\phi^{1}, \ldots, \phi^{n-1}\right\}$ defines a basis of $I^{(1)}$, and $\alpha$ denotes a complement of $I^{(1)}$ in I, the relation

$$
(d \alpha)^{2} \wedge \alpha \wedge \phi^{1} \wedge \cdots \wedge \phi^{n-1} \neq 0
$$

implies that $I^{(1)}$ is completely integrable.

Proof. Equation (9) proves that

$$
\rho\left(I^{(1)}\right) \geqq 2
$$

and since $p_{N}=1$, Theorem 2 applies, and we deduce

$$
\rho\left(I^{(1)}\right) \leqq 0
$$

which is equivalent to the complete integrability of $I^{(1)}$.

This result can be rephrased to give another example of the nonarbitrariness of type which is not included in Proposition 1. 
COROLlaRy 2. Let I denote an n-dimensional Pfaffian system whose type satisfies

$$
p_{N}=1 \text { and } p_{N+1}>2
$$

then I has derived length $N=1$.

Proof. A calculation proves that the class of such a Pfaffian system is

$$
2 \rho(I)+n \text {. }
$$

Hence $p_{N+1}>2$ if and only if $\rho(I) \geqq 2$. Now $I$ has derived length $N=1$ if and only if

$$
I^{(2)}=I^{(1)} \text {, }
$$

which occurs if and only if $I^{(1)}$ is completely integrable. The corollary now follows from Corollary 1.

In particular the Pfaffian systems whose type satisfies $p_{N}=1$ and which have a derived length $N>1$ are all Pfaffian systems of codimension two.

As a final application we prove a result related to the theorem of Cartan in $[2, \S 45$, p. 60].

COROllary 3. Let I be an n-dimensional Pfaffian system whose type satisfies $p_{N}=2$, then if the rank

$$
\rho(I) \geqq 3
$$

and the derived length $N>1$, there exists an intrinsically defined $(n-1)$-dimensional subsystem of codimension two.

Proof. Theorem 2 applies and we deduce

$$
\rho\left(I^{(1)}\right) \leqq 1 \text {. }
$$

In particular every $\phi \in I^{(1)}$ has an exterior derivative of the form

$$
d \phi \equiv \tau \wedge \alpha \bmod I^{(1)}
$$

where $\alpha \in I$ and $\alpha \not \equiv 0 \bmod I^{(1)}$. Applying the exterior derivative to equation (10) yields

$$
\tau \wedge d \alpha \equiv 0 \bmod I
$$

which, if $\tau \not \equiv 0 \bmod I$, implies

$$
(d \alpha)^{2} \equiv 0 \bmod I
$$

Now if $d \alpha^{1}$ and $d \alpha^{2}$ were any two linearly independent elements of $d I \bmod I$ satisfying (11), then

$$
\left(a d \alpha^{1}+b d \alpha^{2}\right)^{3} \equiv 0 \bmod I
$$

for any $a, b \in c(M)$. But $p_{N}=2$, and hence $d \alpha^{1}$ and $d \alpha^{2}$ would form a basis of $d I \bmod I$, and (12) would contradict that $\rho(I) \geqq 3$. 
Therefore, there exists an intrinsically defined $(n-1)$-dimensional subspace $P$ of $I$ such that $\phi \in P$ if and only if

$$
(d \phi)^{2} \equiv 0 \bmod I .
$$

In particular $I^{(1)} \subset P$, and we may choose $\beta^{1}$ a complement of $I^{(1)}$ in $P$. Since $\beta^{1}$ satisfies (13), there exist one-forms $\pi^{1}$ and $\pi^{2}$ complementary to $I$ such that

$$
d \beta^{1} \equiv \pi^{1} \wedge \pi^{2} \quad \bmod I
$$

We have seen that every element $\phi \in I^{(1)}$ is written

$$
d \phi \equiv \tau \wedge \beta^{1} \bmod I^{(1)},
$$

but, since $\tau \wedge d \beta^{1} \equiv 0 \bmod I$, equation (14) gives

$$
\tau \wedge \pi^{1} \wedge \pi^{2} \equiv 0 \bmod I \text {. }
$$

Therefore, if $\left\{\phi^{1}, \ldots, \phi^{n-2}\right\}$ is a basis of $I^{(1)}$ we have $\left\{\phi^{1}, \ldots, \phi^{n-2}, \beta^{1}\right\}$ a basis of $P$ and equations

$$
\begin{aligned}
& d \beta^{1} \equiv \pi^{1} \wedge \pi^{2} \quad \bmod I
\end{aligned}
$$

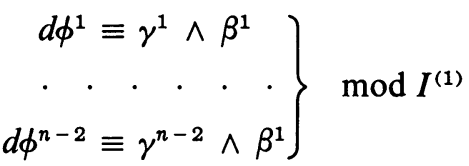

and $\gamma^{i} \wedge \pi^{1} \wedge \pi^{2} \equiv 0 \bmod I$.

If we can prove that these congruences $\bmod I$ can be replaced by congruences $\bmod P$, then

$$
\text { class } P=n+2 \text {. }
$$

Let $\beta^{2}$ define a complement of $P$ in $I$, if $\beta^{2}$ were in the Cartan system $C(P)$, then

$$
d \beta^{2} \wedge \phi^{1} \wedge \cdots \wedge \phi^{n-2} \wedge \beta^{1} \wedge \beta^{2} \wedge \pi^{1} \wedge \pi^{2}=0
$$

by the complete integrability of $C(P)$, and this would imply

$$
\left(d \beta^{2}\right)^{3} \equiv 0 \bmod I
$$

which contradicts the hypothesis. Therefore $\beta^{2} \notin C(P)$ and the congruences mod $I$ may be replaced by congruences mod $P$ as claimed.

Thus the classification of Pfaffian systems whose type satisfies $P_{N}=1$ or 2 leads to Pfaffian systems of codimension two. We will consider the discouraging problem of classifying the Pfaffian systems of codimension two later in this paper.

4. Intrinsic conformal tensors. Let $I$ be an $n$-dimensional Pfaffian system of class $n+2 r$, then we may use the existence of the Cartan system to define an intrinsic symmetric conformal tensor of degree $r$ on $I$ which for $\left\{\phi_{1}^{i}, \ldots, \phi_{r}^{i}\right\} \in I$ is written

$$
\left\langle\phi_{1}^{i}, \ldots, \phi_{r}^{i}\right\rangle
$$


and a representative for which is defined by

$$
d \phi_{1}^{i} \wedge \cdots \wedge d \phi_{r}^{i} \wedge w^{1} \wedge \cdots \wedge w^{n}=\left\langle\phi_{1}^{i}, \ldots, \phi_{r}^{i}\right\rangle \Omega_{n+2 r}
$$

where $\left\{w^{1}, \ldots, w^{n}\right\}$ defines a basis for $I$, and $\Omega_{n+2 r}$ is any nonzero $(n+2 r)$-form in $\Lambda^{n+2 r} C(I)$.

The definition makes sense since any $(n+2 r)$-form in $\Lambda^{n+2 r} C(I)$ is a multiple of $\Omega_{n+2 r}$ and

$$
\left\langle\phi_{1}^{i}, \ldots, \phi_{r}^{i}\right\rangle
$$

is clearly symmetric and $r$-multilinear. If we chose any other basis for $I$, or any other nonzero form in $\Lambda^{n+2 r} C(I)$, then the definition would only be modified by a nonzero function. Since these are the only ambiguities in the definition, the tensor is conformally defined over $c(M)$.

Similar constructions may be used on Pfaffian systems satisfying particular type restrictions. For example, let $I$ be an $n$-dimensional Pfaffian system with type

$$
\left(p_{0}, \ldots, p_{N-2}, m, r, m\right)
$$

then we may define on $I^{(2)}$, the second derived system, an intrinsic symmetric conformal tensor of degree $m$ which for $\left\{\phi_{1}^{i}, \ldots, \phi_{m}^{i}\right\} \in I^{(2)}$ is written

$$
\left\langle\phi_{1}^{i}, \ldots, \phi_{m}^{i}\right\rangle
$$

and a representative for which is defined by

$$
d \phi_{1}^{i} \wedge \cdots \wedge d \phi_{m}^{i} \wedge \alpha^{1} \wedge \cdots \wedge \alpha^{r} \wedge \phi^{1} \wedge \cdots \wedge \phi^{n-2 m-r}=\left\langle\phi_{1}^{i}, \ldots, \phi_{m}^{i}\right\rangle \Omega_{n+m}
$$

where $\left\{\phi^{1}, \ldots, \phi^{n-m-r}\right\}$ defines a basis for $I^{(2)}$, and $\left\{\alpha^{1}, \ldots, \alpha^{r}\right\}$ defines a basis for any complement of $I^{(1)}$ in $I$, and $\Omega_{n+m}$ is any nonzero $(n+m)$-form in $\Lambda^{n+m} C(I)$.

In order to verify that this tensor is intrinsic it suffices to prove that the definition is independent of the choice of complement of $I^{(1)}$ in $I$. Analytically this is equivalent to showing that, for $\left\{\phi_{1}^{i}, \ldots, \phi_{m}^{i}\right\} \in I^{(2)}$ and $\beta$ any element of a complement of $I^{(2)}$ in $I^{(1)}$,

$$
d \phi_{1}^{i} \wedge \cdots \wedge d \phi_{m}^{i} \wedge \beta \equiv 0 \bmod I^{(2)} .
$$

Let $\left\{\beta^{1}, \ldots, \beta^{m}\right\}$ define a basis for any complement of $I^{(2)}$ in $I^{(1)}$, then, if $\phi \in I^{(2)}$, the defining relations for $I^{(2)}$ guarantee the existence of one-forms $\eta_{a}$ such that

$$
d \phi \equiv \sum_{a=1}^{m} \beta^{a} \eta_{a} \bmod I^{(2)}
$$

As a result the left-hand side of $(15)$ is a $(2 m+1)$-monomial involving $(m+1)$ linear differential forms from an $m$-dimensional space, and hence must vanish.

5. Pfaffian systems of codimension four. Let $I$ be a Pfaffian system of codimension four, then the results of $\S 4$ apply which we state as a proposition. 
Proposition 5. Let I be a Pfaffian system of codimension four, then the $c(M)$ module I admits an intrinsically defined symmetric conformal $c(M)$-bilinear form $\langle$,$\rangle . If \left\{\phi^{1}, \ldots, \phi^{n}\right\}$ is a basis of $I$, and $\Omega_{n+4}$ is any nonzero $(n+4)$-form in $\Lambda^{n+4} C(I)$, and $\phi, \hat{\phi} \in I$, then a representative for $\langle$,$\rangle is defined by$

$$
d \phi \wedge d \hat{\phi} \wedge \phi^{1} \wedge \cdots \wedge \phi^{n}=\langle\phi, \hat{\phi}\rangle \Omega_{n+4} .
$$

We recall that the rank of a bilinear form is the dimension of the largest submodule on which the form is nondegenerate, and the index of a bilinear form is the dimension of the largest submodule on which the form is negative definite. We define the rank of the above conformal bilinear form to be the rank of any representative, and the index to be the index of a representative for which the orientation of $I$ and $\Omega_{n+4}$ is so chosen that its index be minimal among all representatives.

Proposition 6. Let I be a Pfaffian system of codimension four, and let $\langle$,$\rangle be$ the intrinsically defined bilinear form of Proposition 5, then the best possible inequalities on its invariants are

and

$$
\text { rank }\langle,\rangle \leqq 6
$$

$$
\text { index }\langle,\rangle \leqq 3 \text {. }
$$

Proof. Visibly the first derived system $I^{(1)}$ is contained in the conjugate subspace of $\langle$,$\rangle on I$, and by equation (2)

$$
\operatorname{dim} I^{(1)} \geqq n-6 .
$$

Therefore, we deduce

$$
\operatorname{rank}\langle,\rangle \leqq 6
$$

Furthermore, since

$$
0 \longrightarrow I^{(1)} \longrightarrow I \stackrel{\delta}{\longrightarrow} d I \bmod I \longrightarrow 0,
$$

the bilinear form $\langle$,$\rangle on I$ induces a bilinear form $\langle,\rangle_{1}$ on $d I \bmod I$ by

for all $\phi, \hat{\phi} \in I$.

$$
\langle\delta(\phi), \delta(\hat{\phi})\rangle_{1}=\langle\phi, \hat{\phi}\rangle
$$

Let $A$ and $B$ be elements in $\Lambda^{2} \Gamma^{*}(M) \bmod I$, and $\alpha$ and $\beta$ any representatives in $\Lambda^{2} \Gamma^{*}(M)$, then we may define a symmetric conformal $c(M)$-bilinear form $\langle,\rangle_{2}$ on $\Lambda^{2} \Gamma^{*}(M) \bmod I$ with a representative defined by

$$
\alpha \wedge \beta \wedge \phi^{1} \wedge \cdots \wedge \phi^{n}=\langle A, B\rangle_{2} \Omega
$$

where $\left\{\phi^{1}, \ldots, \phi^{n}\right\}$ is a basis for $I$, and $\Omega$ is any nonzero $(n+4)$-form in $\Lambda^{n+4} C(I)$.

There is a canonical injection of $c(M)$-modules

$$
0 \rightarrow d I \bmod I \rightarrow \Lambda^{2} \Gamma^{*}(M) \bmod I
$$

and by definition we see that $\langle,\rangle_{1}$ is the restriction of $\langle,\rangle_{2}$. 
Now let $\left\{\pi^{1}, \pi^{2}, \pi^{3}, \pi^{4}\right\}$ define a complement of $I$ in $C(I)$, then

$$
\begin{aligned}
& f^{1}=\pi^{1} \wedge \pi^{2}, \quad f^{2}=\pi^{1} \wedge \pi^{4}, \quad f^{3}=\pi^{1} \wedge \pi^{3}, \\
& f^{4}=\pi^{3} \wedge \pi^{4}, \quad f^{5}=\pi^{2} \wedge \pi^{3}, \quad f^{6}=\pi^{4} \wedge \pi^{2}
\end{aligned}
$$

represents a basis of $\Lambda^{2} \Gamma^{*}(M) \bmod I$, and if we choose

$$
\Omega=\pi^{1} \wedge \pi^{2} \wedge \pi^{3} \wedge \pi^{4} \wedge \phi^{1} \wedge \cdots \wedge \phi^{n}
$$

the matrix of $\left\langle f^{i}, f^{j}\right\rangle_{2}$ is

$$
\left(\begin{array}{c|c}
0_{3} & I_{3} \\
\hline I_{3} & 0_{3}
\end{array}\right)
$$

where $0_{3}$ is the $3 \times 3$ zero matrix and $I_{3}$ is the $3 \times 3$ identity matrix. Since this matrix has index three, and $\langle,\rangle_{1}$ is the restriction of $\langle,\rangle_{2}$ we may only assert

$$
\text { index }\langle,\rangle \leqq 3 \text {. }
$$

As an application of this bilinear form we consider the Pfaffian system associated with a second order partial differential equation in two independent and one dependent variables. Let $J^{r}(2,1)$ denote the fiber bundle of $r$-jets of mappings of the plane $R^{2}$ into the real line $R^{(9)}$.

If $f: R^{2} \rightarrow R$, then the $r$-jet of $f$ at $p \in R^{2}$ is written

$$
j_{p}^{r}(f)
$$

and we may define the $r$-graph of $f$

$$
j^{r}(f): R^{2} \rightarrow J^{r}(2,1)
$$

by

$$
j^{r}(f)(p)=j_{p}^{r}(f) .
$$

We introduce cartesian coordinates $\{x, y\}$ in $R^{2}, z$ in $R$,

$$
p=\partial z / \partial x, \quad q=\partial z / \partial y
$$

in the fibers of $J^{1}(2,1)$ over $R^{2} \times R$, and

$$
r=\partial^{2} z / \partial x^{2}, \quad s=\partial^{2} z / \partial x \partial y, \quad t=\partial^{2} z / \partial y^{2}
$$

in the fibers of $J^{2}(2,1)$ over $J^{1}(2,1)$. Matters being so, a second order partial differential equation is a hypersurface

$$
F(x, y, z, p, q, r, s, t)=0
$$

transversal to the fibers of $J^{2}(2,1)$ over $J^{1}(2,1)$.

There is a canonical Pfaffian system defined on $J^{2}(2,1)$ by

$$
\Omega^{2}(2,1)=\left\{w \in \Gamma^{*} J^{2}(2,1) \mid j^{2}(f)^{*} w=0, \text { for all } f: R^{2} \rightarrow R\right\} .
$$


This system has generators in the above local coordinates given by

$$
\begin{aligned}
& w^{1}=d z-p d x-q d y, \\
& w^{2}=d p-r d x-s d y, \\
& w^{3}=d q-s d x-t d y .
\end{aligned}
$$

The Pfaffian system $I$ associated to a second order partial differential equation $(17)$ is the Pfaffian system on $J^{2}(2,1)$ with generators

$$
I=\left\{w^{1}, w^{2}, w^{3}, d F\right\} .
$$

This is a four-dimensional Pfaffian system of class eight, and as such admits a bilinear form defined for $\phi, \hat{\phi} \in I$ by

$$
d \phi \wedge d \hat{\phi} \wedge w^{1} \wedge w^{2} \wedge w^{3} \wedge d F=\langle\phi, \hat{\phi}\rangle \Omega_{8}
$$

where we choose

$$
\Omega_{8}=-\frac{1}{2} d x \wedge d y \wedge d z \wedge d p \wedge d q \wedge d r \wedge d s \wedge d t .
$$

The forms $w^{1}$ and $d F$ are in the conjugate subspace of $\langle,>$ and

$$
\left(\begin{array}{ll}
\left\langle w^{2}, w^{2}\right\rangle & \left\langle w^{2}, w^{3}\right\rangle \\
\left\langle w^{3}, w^{2}\right\rangle & \left\langle w^{3}, w^{3}\right\rangle
\end{array}\right)=\left(\begin{array}{cc}
\partial F / \partial t & -\frac{1}{2} \partial F / \partial s \\
-\frac{1}{2} \partial F / \partial s & \partial F / \partial r
\end{array}\right) .
$$

Let

$$
\Delta=\partial F / \partial r \partial F / \partial t-\frac{1}{4} \partial F / \partial s^{2},
$$

then the bilinear form $\langle$,$\rangle may have$

(a) Rank one, which means

$$
\Delta=0
$$

or the equation was of parabolic type, and there exists a unique three-dimensional maximal totally isotropic subspace [6] $N$ of $\langle$, $\rangle$ on $I$.

(b) Rank two, and index one, which means

$$
\Delta<0
$$

or the equation was of hyperbolic type, and there exist two three-dimensional maximal totally isotropic subspaces $M_{1}$ and $M_{2}$ of $\langle$,$\rangle on I$.

(c) Rank two and index zero, which means

$$
\Delta>0
$$

or the equation was of elliptic type.

In particular the intrinsic bilinear form gives rise to a definition of type of a second order partial differential equation which is obviously invariant under prolonged contact transformations. 
We will now show that the bilinear form may also be used to define the characteristic curves of such an equation.

Let

$$
f: R^{2} \rightarrow R
$$

be a solution of the equation (17), and $V\left(R^{2}\right)$ the vector field system of all vector fields on $R^{2}$, then we may introduce an injection

defined by

$$
0 \longrightarrow V\left(R^{2}\right) \stackrel{\rho_{f}}{\longrightarrow} I
$$

$$
\rho_{f}(X)=\mathscr{L}_{j^{2}(f) \cdot x} w^{1}
$$

where the right-hand side is the Lie derivative of the one-form $w^{1}$ along the vector field $j^{2}(f)_{*} X$.

This injection induces a symmetric conformal $C\left(R^{2}\right)$-bilinear form $\langle$,$\rangle , on$ $V\left(R^{2}\right)$ by

Since

$$
\langle X \cdot Y\rangle_{f}(p)=\left\langle\rho_{f}(X), \rho_{f}(Y)\right\rangle\left(j_{p}^{2}(f)\right)
$$

$$
\rho_{f}(\partial / \partial x)=w^{2} \quad \text { and } \quad \rho_{f}(\partial / \partial y)=w^{3},
$$

if $I$ has one of the three-dimensional maximal totally isotropic subspaces

$$
N, M_{1}, M_{2},
$$

then there is induced a one-dimensional maximal totally isotropic vector field system in $V\left(R^{2}\right)$ which we denote respectively by

$$
N_{f}, M_{1 f}, M_{2 f}
$$

The annihilators

$$
N_{f}^{\perp}, M_{1 f}^{\perp}, M_{2 f}^{\perp},
$$

are all one-dimensional Pfaffian systems of class at most two, and hence are completely integrable. In any one of these cases if

$$
d \phi=\partial \phi / \partial x d x+\partial \phi / \partial y d y
$$

defines an exact basis, then the vector field

$$
X=\partial \phi / \partial y \partial / \partial x-\partial \phi / \partial x \partial / \partial y
$$

defines the original vector field system. The condition that $X$ define a maximal totally isotropic subspace of $\langle,\rangle_{f}$ on $V\left(R^{2}\right)$ is simply $\langle X, X\rangle_{f}=0$.

Now if

$$
G: J^{2}(2,1) \rightarrow R \text { and } f: R^{2} \rightarrow R
$$

we define $G_{f}: R^{2} \rightarrow R$ by

$$
G_{f}(p)=G \circ j^{2}(f)(p)
$$


With this convention

$$
\langle X, X\rangle_{f}=\partial F / \partial t_{f}(\partial \phi / \partial y)^{2}+\partial F / \partial s_{f} \partial \phi / \partial x \partial \phi / \partial y+\partial F / \partial r_{f}(\partial \phi / \partial x)^{2} .
$$

Thus the first integrals of any of the Pfaffian systems

$$
N_{f}^{\perp}, M_{1 f}^{\perp}, M_{2 f}^{\perp},
$$

are solutions of a quadratic first order partial differential equation. The curves

$$
\phi=\text { constant }
$$

are called the characteristic curves on the source, and the curves

$$
z=\left.f(x, y)\right|_{\phi=\text { constant }}
$$

are called the characteristic curves on the integral surface.

The study of the Pfaffian systems

$$
N, M_{1}, M_{2},
$$

lead to a coordinate free description of the methods of integration of Monge and Ampere which I hope to present in a forthcoming paper.

6. Pfaffian systems of codimension two. Let $I$ be an $n$-dimensional Pfaffian system of class $n+2$ defined on an $(n+2)$-dimensional manifold $M$. Equation (2) implies

$$
\operatorname{dim} I^{(1)} \geqq n-1,
$$

and, if $\operatorname{dim} I^{(1)}=n$, then

$$
I=I^{(1)}
$$

which implies that $I$ is completely integrable and the derived length $N=0$. As a result we will assume that

$$
\operatorname{dim} I^{(1)}=n-1 \text {. }
$$

The inequalities of Proposition 1 then give

$$
\operatorname{dim} I^{(2)} \geqq n-3 \text {. }
$$

If $\operatorname{dim} I^{(2)}=n-1$, then

$$
I^{(1)}=I^{(2)}
$$

which implies that $I^{(1)}$ is completely integrable and the derived length $N=1$.

If $\operatorname{dim} I^{(2)}=n-2$, then Corollary 1 to Theorem 2 applies to $I^{(1)}$, and either $I^{(2)}$ is completely integrable or $I^{(1)}$ is itself a Pfaffian system of codimension two.

As a result the Pfaffian systems of codimension two and derived length $N>1$ are partitioned.into two classes. 
Class (A). The type is of the form

$$
\left(p_{0}, 1, \ldots, 1,2\right)
$$

where $I^{(r)}$ for $1 \leqq r \leqq N-1$ are all Pfaffian systems of codimension two, and $I^{(N)}$ is a $p_{0}$-dimensional completely integrable system. These have been called special systems by Goursat [8], and a theorem due to von Weber asserts that there exist $\left(N+p_{0}+2\right)$-independent functions

$$
\left\{x, z_{1}, \ldots, z_{p_{0}}, y, y_{1}, \ldots, y_{N}\right\}
$$

such that locally $I$ has a basis

$$
\left\{d z_{1}, \ldots, d z_{p_{0}}, d y-y_{1} d x, d y_{1}-y_{2} d x, \ldots, d y_{N-1}-y_{N} d x\right\} .
$$

Class (B). The type has the form $\left(p_{0}, \ldots, p_{N-m}, 2,1, \ldots, 1,2\right)$ where $I^{(r)}$ for $1 \leqq r \leqq m$ are all Pfaffian systems of codimension two, and

$$
P_{N-m+1}=\operatorname{dim} I^{(m-1)}-\operatorname{dim} I^{(m-2)}=2 .
$$

These have been called normal systems by Cartan [4], and have not been studied except for Cartan's paper [3], which deals with Pfaffian systems of type $(0,2,1,2)$.

We thus turn our attention to the normal Pfaffian systems of codimension two. These all contain a derived system $I$ which has type of the form

$$
\left(p_{0}, \ldots, p_{N-2}, 2,1,2\right) \text {. }
$$

Let $\left\{\phi^{1}, \ldots, \phi^{n-3}, \beta^{1}, \beta^{2}, \alpha, \tau, \Delta\right\}$ denote any basis of the Cartan system $C(I)$ such that $\left\{\phi^{1}, \ldots, \phi^{n-3}\right\}$ defines a basis of $I^{(2)},\left\{\beta^{1}, \beta^{2}\right\}$ defines a basis of a complement of $I^{(2)}$ in $I^{(1)}, \alpha$ defines a complement of $I^{(1)}$ in $I$, and $\{\tau, \Delta\}$ defines a basis of a complement of $I$ in $C(I)$. We will call such a basis an adapted basis.

In particular the results of paragraph 4 apply which we state as a proposition.

Proposition 7. Let I be an n-dimensional Pfaffian system of codimension two and type

$$
\left(p_{0}, \ldots, p_{N-2}, 2,1,2\right)
$$

then the $c(M)$-module $I^{(2)}$ admits an intrinsically defined symmetric conformal $c(M)$-bilinear form $\langle$, $\rangle$. If

$$
\left\{\phi^{1}, \ldots, \phi^{n-3}, \beta^{1}, \beta^{2}, \alpha, \tau, \Delta\right\}
$$

is any adapted basis for $C(I), \Omega_{n+2}$ is a nonzero $(n+2)$-form in $\Lambda^{n+2} C(I)$, then a representative for $\langle$,$\rangle is defined for \phi, \hat{\phi} \in I^{(2)}$ by

$$
d \phi \wedge d \hat{\phi} \wedge \alpha \wedge \phi^{1} \wedge \cdots \wedge \phi^{n-3}=\langle\phi, \widehat{\phi}\rangle \Omega_{n+2} .
$$

Now by definition of the first derived system we will have relations

$$
\begin{aligned}
& d \beta^{1} \equiv(a \tau+b \Delta) \wedge \alpha \\
& d \beta^{2} \equiv(c \tau+e \Delta) \wedge \alpha
\end{aligned}
$$


and if $(a \tau+b \Delta) \wedge(c \tau+e \Delta)=(a e-b c) \tau \wedge \Delta=0$, then there would exist functions $\lambda$ and $\mu$ such that

$$
\lambda d \beta^{1}-\mu d \beta^{2} \equiv 0 \bmod I^{(1)},
$$

and this would contradict the fact that $\beta^{1}$ and $\beta^{2}$ define a basis for a complement of $I^{(2)}$ in $I^{(1)}$. Thus

$$
a e-b c \neq 0,
$$

and by applying a nonsingular transformation to $\beta^{1}$ and $\beta^{2}$ we may assume that

$$
\begin{aligned}
& d \beta^{1} \equiv \tau \wedge \alpha \\
& d \beta^{2} \equiv \Delta \wedge \alpha
\end{aligned}
$$

An adapted basis which satisfies the additional conditions (18) will be called a normalized adapted basis.

The integrability conditions for $I^{(2)}$ in $I^{(1)}$ give

$$
d \phi^{i} \wedge \beta^{1} \wedge d \beta^{2}-d \phi^{i} \wedge \beta^{2} \wedge d \beta^{1} \equiv 0 \bmod I^{(2)}
$$

and by (16) this becomes

$$
\beta^{2} \wedge \eta_{2}^{i} \wedge \beta^{1} \wedge d \beta^{2}-\beta^{1} \wedge \eta_{1}^{i} \wedge \beta^{2} \wedge d \beta^{1} \equiv 0 \bmod I^{(2)}
$$

and by (18) this gives

$$
\beta^{2} \wedge \eta_{2}^{i} \wedge \beta^{1} \wedge \Delta \wedge \alpha-\beta^{1} \wedge \eta_{1}^{i} \wedge \beta^{2} \wedge \tau \eta \alpha \equiv 0 \bmod I^{(2)}
$$

Therefore

$$
\eta_{1}^{i} \wedge \tau+\eta_{2}^{i} \wedge \Delta \equiv 0 \bmod I,
$$

and Cartan's lemma implies the existence of functions $f^{i}, g^{i}, h^{i} \in c(M)$ such that

$$
\left(\begin{array}{l}
\eta_{1}^{i} \\
\eta_{2}^{i}
\end{array}\right) \equiv\left(\begin{array}{ll}
f^{i} & g^{i} \\
g^{i} & h^{i}
\end{array}\right)\left(\begin{array}{l}
\tau \\
\Delta
\end{array}\right) \quad \bmod I .
$$

We now introduce the vector notation

$$
\beta=\left(\beta^{1}, \beta^{2}\right): \eta^{i}=\left(\eta_{1}^{i}, \eta_{2}^{i}\right): \tau={ }^{t}(\tau, \Delta)
$$

and hence equation (16) becomes

$$
d \phi^{i} \equiv\left(\beta, \eta^{i}\right) \bmod I^{(2)}
$$

where $($,$) is a standard euclidean inner product, and equation (18) becomes$

$$
d \beta \equiv \tau \wedge \alpha \bmod I^{(2)}
$$

and equation (19) becomes

$$
\eta^{i} \equiv F^{i} \tau \bmod I
$$

where we have written

$$
F^{i}=\left(\begin{array}{ll}
f^{i} & g^{i} \\
g^{i} & h^{i}
\end{array}\right)
$$


Thus given any normalized adapted basis of $C(I)$, we have constructed a $2 \times 2$ symmetric matrix from every element of $I^{(2)}$.

Let $W$ denote the set of $2 \times 2$ symmetric matrices with entries from $c(M)$, viewed as a $c(M)$-module under addition and scalar multiplication, then this construction defines a $c(M)$-module homomorphism

$$
\pi: I^{(2)} \rightarrow W
$$

where, for $\phi \in I^{(2)}$ satisfying

$$
d \phi \equiv(\beta, \eta) \bmod I^{(2)}
$$

and

$$
\eta \equiv F \tau \bmod I
$$

with respect to a normalized adapted basis, we set

$$
\pi(\phi)=F \text {. }
$$

The definition of the homomorphism is not intrinsic, but only depends on a choice of basis $\tau$ for a complement of $I$ in $C(I)$.

The invertible $2 \times 2$ matrices with entries from $c(M)$ form a group which we denote by $\mathrm{Gl}(2, c(M))$, and if $Q \in \mathrm{Gl}(2, c(M))$ and we choose a new basis $\tau^{\prime}$ for a complement of $I$ in $C(I)$ with

$$
\tau^{\prime} \equiv Q \tau \quad \bmod I
$$

and let $\pi_{Q}$ denote the above homomorphism computed with respect to any normalized adapted basis with $\tau^{\prime}$ the complement of $I$ in $C(I)$, then

where

$$
\pi_{Q}(\phi)=\rho(Q) \pi(\phi)
$$

is the action defined by

$$
\rho: \mathrm{G} 1(2, c(M)) X W \rightarrow W
$$

$$
\rho(Q) F={ }^{t} Q F Q
$$

for $Q \in \mathrm{G} 1(2, c(M))$, and $F \in W$. This proves

Proposition 8. Let I be an n-dimensional Pfaffian system with type

$$
\left(p_{0}, \ldots, p_{N-2}, 2,1,2\right)
$$

then every element $\phi$ in the second derived system $I^{(2)}$, has two invariants

$$
\text { rank } \pi(\phi) \text { and index } \pi(\phi) \text {. }
$$

Next, we observe that all the homomorphisms $\pi_{Q}$ differ by at most an automorphism of $W$, and as a result their kernels coincide and define an intrinsically defined subspace $S$. Let us remark that

$$
I^{(3)} \subset S
$$

because if $\phi \in I^{(3)}$, then $d \phi \equiv 0 \bmod I^{(2)}$ and $\pi(\phi)=0$. 
The classical invariant theory of nets of binary quadrics suggests a symmetric $c(M)$-bilinear form $\langle,\rangle_{L}$ for $W$, where for

$$
F=\left(\begin{array}{ll}
f & g \\
g & h
\end{array}\right) \text { and } G=\left(\begin{array}{ll}
r & s \\
s & t
\end{array}\right)
$$

elements of $W$, we define

$$
\langle F, G\rangle_{L}=f t+h r-2 g s .
$$

By choosing the basis $\left(F^{1}, F^{2}, F^{3}\right)$ where

$$
F^{1}=\left(\begin{array}{ll}
1 & 0 \\
0 & 1
\end{array}\right) \quad F^{2}=\left(\begin{array}{rr}
1 & 0 \\
0 & -1
\end{array}\right) \quad F^{3}=\left(\begin{array}{ll}
0 & 1 \\
1 & 0
\end{array}\right)
$$

the matrix $\left\langle F^{i}, F^{j}\right\rangle_{L}$ becomes

$$
\left(\begin{array}{rrr}
2 & 0 & 0 \\
0 & -2 & 0 \\
0 & 0 & -2
\end{array}\right)
$$

which proves that $\langle,\rangle_{L}$ is nondegenerate and Lorentzian.

This bilinear form has the additional property that for $Q \in \mathrm{G} 1(2, c(M))$

$$
\left\langle{ }^{t} Q F Q,{ }^{t} Q G Q\right\rangle_{L}=(\operatorname{det} Q)^{2}\langle F, G\rangle_{L} .
$$

We will let

$$
\langle,\rangle_{C(L)}
$$

denote the conformal $C(M)$-bilinear form with representative $\langle,\rangle_{L}$.

Proposition 9. Let $\pi: I^{(2)} \rightarrow W$ be the $c(M)$-module homomorphism constructed with respect to any normalized adapted basis, then for $\phi, \hat{\phi} \in I^{(2)}$ we have

$$
\langle\phi, \hat{\phi}\rangle=\langle\pi(\phi), \pi(\hat{\phi})\rangle_{C(L)}
$$

Proof. By equation (16)

$$
d \phi^{i} \wedge d \phi^{j} \equiv\left(\eta_{2}^{i} \wedge \eta_{1}^{j}-\eta_{1}^{i} \wedge \eta_{2}^{j}\right) \wedge \beta^{1} \wedge \beta^{2} \bmod I^{(2)}
$$

letting

$$
\left(\begin{array}{l}
\eta_{1}^{i} \\
\eta_{2}^{i}
\end{array}\right)=\left(\begin{array}{ll}
f^{i} & g^{i} \\
g^{i} & h^{i}
\end{array}\right)\left(\begin{array}{l}
\tau \\
\Delta
\end{array}\right) \bmod I
$$

we have

$$
\left(\eta_{1}^{i} \wedge \eta_{2}^{j}-\eta_{2}^{i} \wedge \eta_{1}^{j}\right) \equiv\left(f^{i} h^{j}+h^{i} f^{j}-2 g^{i} g^{j}\right) \tau \wedge \Delta \bmod I
$$

and hence

$$
\left\langle\phi^{i}, \phi^{j}\right\rangle=\left\langle F^{i}, F^{j}\right\rangle_{C(L)}=\left\langle\pi\left(\phi^{i}\right), \pi\left(\phi^{j}\right)\right\rangle_{C(L)}
$$

as was to be proved. 
Let us assume that $\langle$,$\rangle is identically zero on I^{(2)}$. Then since $\pi: I^{(2)} \rightarrow W$ is an isometry, $I^{(2)}$ must map into a maximal totally isotropic subspace of $W$, and hence

$$
\operatorname{dim} I^{(2)}-\operatorname{dim} S \leqq 1 .
$$

As a special case it may happen that $\operatorname{dim} I^{(2)}=\operatorname{dim} S$ which implies that $\pi$ is the zero map.

THEOREM 3. Let I be an n-dimensional Pfaffian system whose type satisfies $p_{N}=1$ and $p_{N-1}=2$; then I has derived length $N=2$ if and only if the homomorphism $\pi$ is the zero map.

Proof. If $I$ has derived length $N=2$, then

$$
I^{(2)}=I^{(3)}
$$

hence $I^{(2)}$ is completely integrable and the Frobenius conditions imply that $\pi$ is the zero map.

Conversely let

$$
\pi: I^{(2)} \rightarrow\{0\}
$$

then, if $\left\{\phi^{1}, \ldots, \phi^{n-3}\right\}$ is a basis of $I^{(2)}$, by equation (16) we have relations

$$
d \phi^{i} \equiv\left(\beta, \eta^{i}\right) \bmod I^{(2)}
$$

and

$$
\eta^{i} \equiv 0 \bmod I
$$

but this implies

$$
C\left(I^{(2)}\right) \subset I
$$

and since the Cartan system $C\left(I^{(2)}\right)$ is completely integrable and $I^{(1)}$ is not completely integrable

$$
C\left(I^{(2)}\right)=I^{(2)}
$$

and the theorem is proved.

Proposition 10. Let I be an n-dimensional Pfaffian system of type

$$
\left(p_{0}, \ldots, p_{N-2}, 2,1,2\right)
$$

then

$$
\text { Class } I^{(2)} \leqq n+1
$$

if and only if

$$
\langle,\rangle \equiv 0 \text { on } I^{(2)} \text {. }
$$

Proof. If Class $I^{(2)} \leqq n+1$, then $\langle,\rangle \equiv 0$ since the defining equations would be an $(n+2)$-form in an $(n+1)$-dimensional space. 
If $\langle,\rangle \equiv 0$ and $I^{(2)}=S$, then we saw that $I^{(2)}$ was completely integrable and hence

$$
\text { Class } I^{(2)}=n-3 \text {. }
$$

If $\langle,\rangle \equiv 0$, and $I^{(2)} \neq S$, then let $\gamma$ denote a complement of $S$ in $I^{(2)}$. Since

$$
\langle\pi(\gamma), \pi(\gamma)\rangle_{L} \equiv 0
$$

we know det $\pi(\gamma)=0$, and hence we may choose a normalized adapted basis

$$
\left(\phi^{1}, \ldots, \phi^{n-3}, \beta^{1}, \beta^{2}, \alpha, \tau, \Delta\right)
$$

such that

$$
\pi(\gamma)=\left(\begin{array}{ll}
1 & 0 \\
0 & 0
\end{array}\right)
$$

and as a result

$$
d \gamma \equiv \beta^{1} \wedge \tau+\left(a_{1} \beta^{1}+a_{2} \beta^{2}\right) \wedge \alpha \bmod I^{(2)}
$$

where $a_{1}, a_{2} \in C(M)$. If $\phi \in S$, then

$$
d \phi \equiv(\beta, \eta) \bmod I^{(2)}
$$

and $\eta \equiv 0 \bmod I$. Therefore, equations (20), and (21) prove that

$$
\text { Class } I^{(2)} \leqq n+1
$$

which completes the proof.

\section{BIBLIOGRAPHY}

1. Elie Cartan, Oeuvres completes, Gauthier-Villars, Paris, 1953.

2. - - Sur l'integration de certains systemes de Pfaff de caractere deux, Oeuvres Completes, Gauthier-Villars, Paris, 1953, pp. 483-502.

3. - Les systemes de Pfaff a cinq variables, Oeuvres Completes, Gauthier-Villars, Paris, 1953, pp. 927-1010.

4. _ Sur l'equivalence absolue de certains systemes d'equations differentielles, Oeuvres Completes, Gauthier-Villars, Paris, 1953, pp. 1133-1141.

5. - Les systemes differentiels exterieurs et leurs applications geometriques, Hermann, Paris, 1945.

6. C. Chevalley, The algebraic theory of spinors, Columbia Univ. Press, New York, 1954.

7. D. Dearborn, Inequalities among the invariants of Pfaffian systems, Duke Math. J. 2 (1936), 705-711.

8. E. Goursat, Leçons sur le probleme de Pfaff, Hermann, Paris, 1962.

9. H. Levine, Singularities of differentiable mappings, Mathematischen Institut der Universitat Bonn, 1959.

10. S. Sternberg, Lectures on differential geometry, Prentice-Hall, Englewood Cliffs, N. J., 1964.

Courant Institute of Mathematical Sciences, New York University, NEW YoRK, NeW YoRK 